\title{
In-Plane Edge Magnetism in Graphene-Like Nanoribbons
}

\author{
S. Krompiewski ${ }^{a *}$ AND G. CUniBERTI ${ }^{b}$
}

${ }^{a}$ Institute of Molecular Physics, Polish Academy of Sciences, M. Smoluchowskiego 17, 60-179 Poznań, Poland ${ }^{b}$ TU Dresden, 01062 Dresden, Germany: Institute for Materials Science and Max Bergmann Center of Biomaterials; Center for Advancing Electronics Dresden; and Dresden Center for Computational Materials Science (DCMS)

This paper is devoted to identification of the most important factors responsible for formation of magnetic moments at edges of graphene-like nanoribbons. The main role is attributed to the Hubbard correlations (within unrestricted Hartree-Fock approximation) and intrinsic spin-orbit interactions, but additionally a perpendicular electric field is also taken into account. Of particular interest is the interplay of the in-plane edge magnetism and the energy band gap. It is shown that, with the increasing electric field, typically the following phases develop: magnetic insulator (with in-plane spins), nonmagnetic narrow-band semiconductor, and nonmagnetic band insulator.

DOI: 10.12693/APhysPolA.131.828

PACS/topics: 75.75.-c, 75.30.Gw, 61.48.Gh

\section{Introduction}

Graphene-like nanostructures constitute a new promising class potentially important for future nanoelectronics and spintronics. Unlike graphene, they usually have sufficiently large intrinsic spin-orbit interaction which strongly impacts their electronic and magnetic properties [1-3]. It is now known that zigzag-shaped edges of graphene and graphene-like nanoribbons (GLNRs) may have noticeable magnetic moments, and their ground state configuration corresponds to the in-plane spin arrangement rather than the out-of-plane one $[4,5]$. The aim of this study is to estimate the effect of both ISOI and perpendicular external electric field on energy gaps and edge magnetic moments of GLNR.

\section{Methodology}

The method is based on a tight-binding Hamiltonian with additional terms describing: intrinsic spin-orbit interaction (ISOI), the Hubbard correlations responsible for either out-of-plane or in-plane magnetic ordering, and perpendicular electric potential $V$. The potential is applied between the sublattice atoms $\mathrm{A}$ and $\mathrm{B}$ which are not coplanar but shifted with respect to each other typically by $c a .0 .5 \AA$. The Hamiltonian reads

$$
\begin{aligned}
H & =\sum_{\langle i, j\rangle, \sigma} t_{i, j} c_{i \sigma}^{+} c_{j \sigma}+\mathrm{i} t_{S O} \sum_{\langle\langle i, j\rangle\rangle} \nu_{i, j}\left(c_{i \uparrow}^{+} c_{j \uparrow}-c_{i \downarrow}^{+} c_{j \downarrow}\right) \\
& +H_{U}+V \sum_{i, \sigma} \mu_{i} c_{i \sigma}^{+} c_{i \sigma}
\end{aligned}
$$

where $H_{U}$ takes the following form for the aforementioned orderings:

$$
\begin{aligned}
& H_{U}^{\text {out }}=U \sum_{i}\left(\left\langle n_{i \downarrow}\right\rangle n_{i \uparrow}+\left\langle n_{i \uparrow}\right\rangle n_{i \downarrow}-\left\langle n_{i \uparrow}\right\rangle\left\langle n_{i \downarrow}\right\rangle\right), \\
& H_{U}^{\text {in }}=-U \sum_{i}\left(\left\langle S_{i}^{+}\right\rangle S_{i}^{-}+\left\langle S_{i}^{-}\right\rangle S_{i}^{+}-\left\langle S_{i}^{+}\right\rangle\left\langle S_{i}^{-}\right\rangle\right) .
\end{aligned}
$$

The first sum in (1) runs over nearest neighbor sites, and

\footnotetext{
*e-mail: stefan@ifmpan.poznan.pl
}

the second one - over next nearest neighbors. $t_{i, j}$ is the hopping integral, $t_{S O}$ - ISOI parameter, $U$ stands for on-site electron repulsion, and

$$
\begin{aligned}
n_{i \sigma} & =c_{i \sigma}^{+} c_{i \sigma}, \quad S_{i}^{+}=c_{i \uparrow}^{+} c_{i \downarrow}, \quad S_{i}^{-}=c_{i \downarrow}^{+} c_{i \uparrow}, \\
\sigma & =\uparrow, \downarrow .
\end{aligned}
$$

Moreover, $\nu_{i j}= \pm 1$ depending on whether the route from the lattice site $i$ to the next neighbor $j$ via their common nearest neighbor $k$ is clockwise or counterclockwise, whereas $\mu_{i}= \pm 1$ for $\mathrm{A}$ and $\mathrm{B}$ sublattice sites, respectively. The angle brackets, in turn, mean the expectation value over the Hamiltonian ground-state. Hereafter $\left\langle n_{i \sigma}\right\rangle$ will be replaced by $\left\langle n_{i \sigma}\right\rangle-\left\langle n_{i \sigma}+n_{i-\sigma}\right\rangle / 2=\left\langle n_{i \sigma}-n_{i-\sigma}\right\rangle / 2$ in order to always keep the charge neutrality point at $E=0$.

The Hubbard parts of the Hamiltonian can be schematically presented as

$$
H^{\text {out }}=\left[\begin{array}{ll}
\uparrow \uparrow & \\
& \downarrow \downarrow
\end{array}\right], \quad H^{\text {in }}=\left[\begin{array}{cc} 
& \uparrow \downarrow \\
\downarrow \uparrow &
\end{array}\right] .
$$

The magnetization is then equal to $M_{i, \text { out }}=\mu_{\mathrm{B}}\left\langle n_{i \uparrow}-n_{i \downarrow}\right\rangle$ and $M_{i \text {,in }}=\mu_{\mathrm{B}}\left\langle S_{i}^{+}+S_{i}\right\rangle$ in the out-of-plane and the in-plane configuration, respectively. The problem is exactly solved by a direct diagonalization of the full Hamiltonian (cf. [5, 6]).

\section{Results}

Figure 1 shows a distribution of magnetic moments in a small rectangular GLNR composed of $W_{a c}=6$ zigzag lines (width), and $L_{z z}=8$ blunt saw-teeth lines (length), the last line is incomplete for symmetry reasons. The sites are numbered column-wise, so the first maximum (and last minimum) in Fig. 1 is due to the edges. It is clearly seen that absolute values of the magnetic moments in the in-plane configuration are usually slightly higher than those corresponding to the out-of-plane configuration. The difference would disappear in the isotropic case of $t_{\text {so }}=0$ [7].

Another effect of interest here is the impact of perpendicular electric field on edge magnetic moments and 


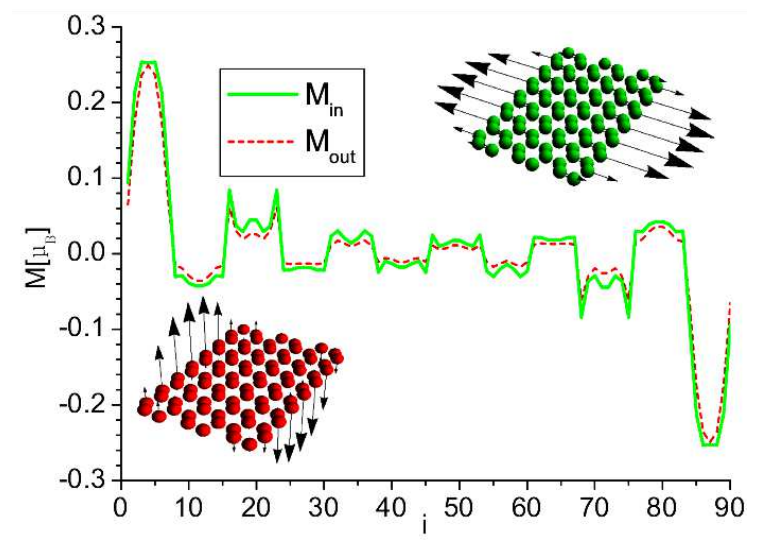

Fig. 1. Local magnetic moments in an exemplary nanoribbon containing 90 lattice sites. The solid (dashed) curve corresponds to the stable in-plane (unstable outof-plane) configuration. The parameters are: $U / t=1$, $t_{\text {so }} / t=0.025, V=0$.

energy levels of the GLNR. Perpendicular electric field effects have been studied, both in graphene bilayers $[8,9]$ as well as in graphene-like buckled systems [10-12] but, to our best knowledge, not for the GLNRs with in-plane edge magnetic moments.

Figure 2 shows that perpendicular staggered voltage $V$ suppresses edge magnetic moments quite strongly. In the in-plane configuration (solid line) the maximum magnetic moment $m_{\max }$ drops rapidly for $\mathrm{eV} / \mathrm{t}$ exceeding 0.07 .

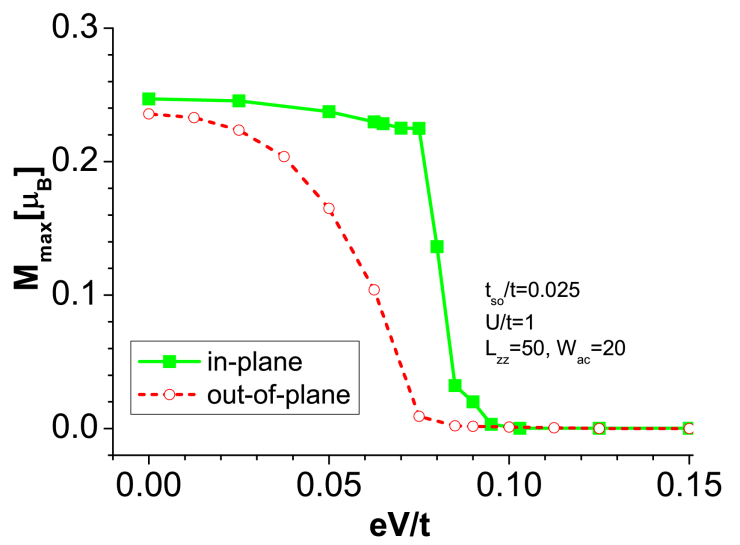

Fig. 2. Impact of the perpendicular voltage on the inplane edge magnetism of the GLNR $L_{z z}=50$ long and $W_{a c}=20$ wide. For comparison, a plot corresponding to the unstable out-of-plane configuration is also shown (dashed curve).

It is also interesting to have a closer look at an energy gap in the case of the stable in-plane configuration. As shown in Fig. 3, it is obvious that the gap is never completely closed by $V$. Moreover, a clear correlation between the edge magnetism and the energy gap is readily seen by comparing Fig. 2 and Fig. 3. In particular, the system behaves as: (i) magnetic insulator for small $V$, then (ii) narrow band-non-magnetic semiconductor, and finally (iii) non-magnetic band insulator for high voltages.

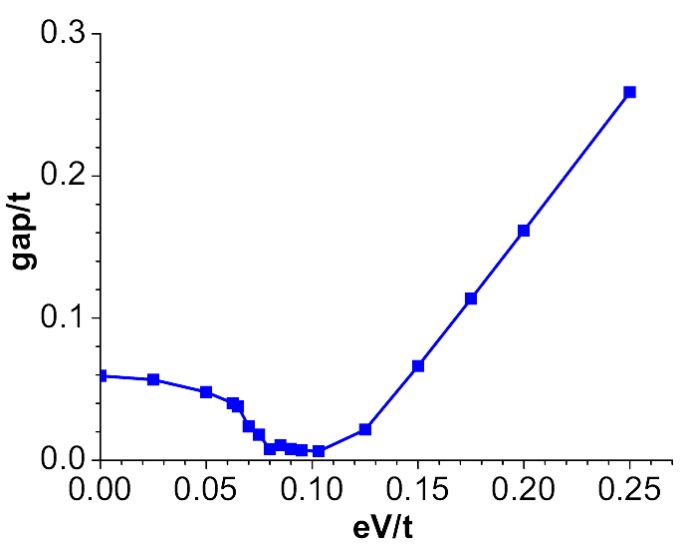

Fig. 3. Energy gap against the perpendicular voltage for the GLNR in the ground state (in-plane magnetic configuration). The parameters are as in Fig. 2.

\section{Conclusions}

Summarizing, it has been shown that graphene-like nanoribbons may reveal quite significant edge magnetic moments, and the energetically favorable arrangement of edge magnetic moments is the in-plane one. The impact of the perpendicular voltage on both energy gaps and the edge magnetism is quite strong. With increasing voltage the nanoribbon changes its electric properties, from magnetic insulator through non-magnetic narrowband semiconductor to non-magnetic band insulator. So in the non-magnetic region the observed behavior resembles that of graphene.

\section{Acknowledgments}

This project was supported by the Polish National Science Centre from funds awarded through the decision No. DEC-2013/10/M/ST3/00488.

\section{References}

[1] G.G. Guzman-Verri, L.C. Lew Yan Voon, Phys. Rev. B 76, 075131 (2007).

[2] M. Ezawa, J. Phys. Soc. Jpn. 84, 121003 (2015).

[3] A.K. Geim, I.V. Grigorieva, Nature 499, 419 (2013).

[4] J.L. Lado, J. Fernandez-Rossier, Phys. Rev. Lett. 113, 027203 (2014).

[5] I. Weymann, J. Barnaś, S. Krompiewski, Phys. Rev. B 92, 045427 (2015).

[6] S. Krompiewski, Nanotechnology 25, 465201 (2014); ibid. 22, 445201 (2011).

[7] K. Wakabayashi, K. Sasaki, T. Nakanishi, T. Enoki, Sci. Technol. Adv. Mater. 11, 054504 (2010).

[8] R. van Geldern, C.M. Smith, Phys. Rev. B 81, 125435 (2010).

[9] A. Dyrdał, J. Barnaś, J. Phys. Condens. 24, 275302 (2012).

[10] N.D. Drummond, V. Zolyomi, V.I. Fal'ko, Phys. Rev. B 85, 075423 (2012).

[11] Z. Ni, Q. Liu, K. Tang, J. Zheng, J. Zhou, R. Qin, Z. Gao, D. Yu, J. Liu, Nano Lett. 12, 113 (2012).

[12] M. Ezawa, New J. Phys. 14, 033003 (2012). 